\title{
A stable nodal integration method for static and quasi-static electromagnetic field computation
}

\author{
Hui Feng ${ }^{\mathrm{a}, \mathrm{b}}$, Xiangyang Cui ${ }^{\mathrm{a}, \mathrm{b}, *}$, Guangyao $\mathrm{Li}^{\mathrm{a}, \mathrm{b}}$ \\ ${ }^{a}$ State Key Laboratory of Advanced Design and Manufacturing for Vehicle Body, Hunan \\ University, Changsha 410082, PR China \\ ${ }^{\mathrm{b}}$ Joint Center for Intelligent New Energy Vehicle, Shanghai, 201804, PR China
}

\begin{abstract}
A stable nodal integration method (SNIM) is presented to solve static and quasi-static electromagnetic problems in this paper. The analysis domain is firstly discretized into a set of triangular or tetrahedral elements, and linear interpolation is adopted within each element. A weakened weak formulation based on the nodes is further considered, framing the so-called node-based smoothing domains. Equivalent smoothing domains are then acquired as circular or spherical regions, where the gradient of shape function is expanded as the first order Taylor form. Subsequently, four or six temporary integration points on the region are picked to obtain items of the stiffness matrix and the external load vector. By simplifying the equations, the stiffness matrix can be received in quite concise form with one point integration and stabilization terms, which are calculated on original node-based smoothing domains. The implementation of SNIM on electromagnetic problems is thus realized. The proposed formulation is validated against both analytical solutions and traditional methods, and its effectiveness and potentialities can be well represented and clarified by numerical examples.
\end{abstract}

Key words: Stable nodal integration method; Computational electromagnetics; Node-based smoothed finite element method; Linear triangular or tetrahedral mesh.

* Corresponding author. Tel: 86-0731-88821717; Fax: 86-731-8822051

Email address: cuixy@hnu.edu.cn (Xiangyang Cui) 


\section{Introduction}

Finite element method (FEM), boundary element method (BEM) and mesh-free methods have been widely developed to solve various types of practical problems of engineering and science. While there is still a growing interest in the development of new advanced methods. Many formulations are becoming popular due to their attractive features. Some meshless mothods, such as element-free Galerkin (EFG) method [1-3], radial basis function (RBF) method [4] and moving least square reproducing kernel method [5], win the favor due to their high adaptivity and low cost to prepare input data for numerical analyses. In recent years, a G space theory was proposed by GR Liu et al $[6,7]$, which forms the foundation of the node-based smoothed finite element method (NS-FEM) [8,9] and a class of numerical methods, such as the node-based smoothed point interpolation method (NS-PIM) [10], smoothed finite element method (SFEM) [11], edge-based smoothed finite element method (ES-FEM) [12] and so on. This class of methods work with weakened-weak forms $\left(\mathrm{W}^{2}\right)$ and use the gradient smoothing operation to approximate the derivatives of the field function in global Galerkin weak forms $[6,7]$. The smoothing operation can effectively softening the model and results in a number of excellent properties, such as good accuracy, high convergence rate, and the insensitivity to element distortion [13]. The gradient smoothing technique was incorporated into the extended finite element method (XFEM) or the phantom-node method by researchers for analyzing fracture and crack growth problems [14-16], and the good accuracy and high convergence rate can also be obtained. 
NS-FEM, as a kind of nodal integration scheme carrying prominent characteristics [17], attracts the attention of the authors. In the early years, actually, attempts have been made to develop nodal integration methods for computation [1, $17,18]$ due to the complexity involved in Gauss integration for Galerkin methods. In 1994, an element-free Galerkin method which is applicable to arbitrary shapes but requires only nodal data was applied to elasticity and heat conduction problems [1]. The paper showed that the method offers high convergence rate and achieves high resolution of localized steep gradients. And it requires no postprocessing for the output of strains and stresses or other field variables which are derivatives of the primary-dependent variables since these quantities are already very smooth. However, this method leads to a numerical instability due to under integration of the weak form and vanishing derivatives of shape functions at the nodes, which definitely hinders it's further implementation and development [18]. A strain smoothing stabilization for nodal integration was proposed $[18,19]$ to eliminate spatial instability in nodal integration. For convergence, an integration constraint (IC) was introduced as a necessary condition for a linear exactness in the mesh-free Galerkin approximation. Results showed that the accuracy and convergent rates in the mesh-free method with a direct nodal integration are improved considerably by the proposed stabilization conforming nodal integration method. NS-FEM is proposed following the stabilized conforming nodal integration scheme $[18,19]$, but it is developed based on FEM theory [17]. So some unique features are captured by NS-FEM: (1) it is usually developed for tetrahedral or triangular elements which can be easily meshed for really 
complicated geometries; (2) linear interpolation is adopted; (3) essential boundary conditions can be directly applied; (4) supporting domain and supporting nodes for each node are naturally formed; (5) it does not contain any parameters to be determined. NS-FEM is simple in theory and implementation, which makes it quite suitable for practical engineering problems.

Studies have demonstrated that NS-FEM performs well in plastic analysis [20], adaptive analysis [21, 22], heat transfer analysis [23] and fracture analysis [24]. However, the "overly-soft" property, which maintains the major difficulty of nodal integration methods [25-30], hinders its application. A stable nodal integration method with gradient smoothing (SNIM-SG) was firstly presented by the authors applied in solid mechanics analysis [31], and have been successfully used to solve acoustic and thermal problems [32] showing worthy properties. This paper is an attempt of the proposed method applying on electromagnetic field. The electromagnetic problems have been studied for decades by numerical methods. A variety of FEMs and meshless methods have been developed and implemented in electromagnetic problems showing their advantages [33-38]. Element-free Galerkin (EFG) method was developed in recent years $[2,3,39-41]$, showing that it is suitable for situations when the remeshing of the analysis domain must be performed. While quadrilateral and hexahedral elements, high order elements, and edge elements are generally adopted in FEMs, and meshless methods mostly use relatively complex functions to approximate field variables. Linear nodal element based on tetrahedral element is rarely adopted in 3D analysis $[42,43]$, and its relative error reaches around ten percent when applied to 
solve benchmark problem 7 of the TEAM workshop [44]. This paper intents to make efforts on the application of linear nodal triangular and tetrahedral elements in electromagnetic fields. Based on previous work by the authors' team, a stable nodal integration method (SNIM) is therefore utilized for static and quasi-static examples, which proved to be successful and encouraging. The application of SNIM on electromagnetic problems shows potentialities to be further studied. Since all information is carried on nodes and the method can solve mechanical and thermal problems successfully, a series of coupled field problems may have a novel solution.

\section{Theoretical basis}

To note that, $3 \mathrm{D}$ problem is discussed in detail in this work, and $2 \mathrm{D}$ formulation can be obtained following the same procedure.

\subsection{Governing equations}

In electromagnetic field analysis, the field variables are typically expressed in terms of potentials, and the governing equations for electrostatic and quasi-static electromagnetic problems are Poisson equation and the diffusion equation respectively.

\subsubsection{Poisson equation}

The Poisson equation with inhomogeneous boundary conditions in Cartesian coordinates can be written as [41]

$$
\begin{array}{cc}
\nabla \cdot \varepsilon \nabla \phi=-\rho & \text { on } \Omega \\
\phi=\phi_{0} & \text { on } S_{1} \\
-\varepsilon \frac{\partial \phi}{\partial n}=h & \text { on } S_{2}
\end{array}
$$


where $\phi$ is the electric scalar potential, $\rho$ is electric charge density, $\varepsilon$ is the material permittivity, $\phi_{0}$ is the prescribed electric potential on boundary $S_{1}$, and $h$ is the prescribed normal derivative of electric potential on $S_{2}$. To note that, regarding the permittivity, the reluctivity and the conductivity, they have all been written into scalar form since this paper mainly deals with linear and isotropic materials, and above parameters of these materials are constants.

\subsubsection{Diffusion equation}

For quasi-static eddy current problem, the diffusion equation in Cartesian coordinates can be expressed as [41]

$$
\begin{gathered}
\nabla \times v \nabla \times \dot{\mathbf{A}}-\nabla(v \nabla \cdot \dot{\mathbf{A}})+j \omega \sigma \dot{\mathbf{A}}+\sigma \nabla \dot{\phi}=\dot{\mathbf{J}}_{s} \\
\nabla \cdot(j \omega \sigma \dot{\mathbf{A}}+\sigma \nabla \dot{\phi})=0
\end{gathered}
$$

where $v$ is the magnetic reluctivity, $\sigma$ is the conductivity, $\dot{\mathbf{A}}$ is the magnetic vector potential, $\dot{\mathbf{J}}_{s}$ is the current density, and $\omega$ is the angular frequency of source current. Dots on $\dot{\mathbf{A}}, \dot{\phi}$ and $\dot{\mathbf{J}}_{s}$ denote them to be complex values. And term $-\nabla(v \nabla \cdot \dot{\mathbf{A}})$ is introduced for the inclusion of Coulomb gauge.

\subsection{Discrete equations by FEM}

\subsubsection{Poisson equation}

Domain in analysis can be discretized into a set of triangular or tetrahedral elements. Within each element, linear interpolation is adopted to approximate the field variable. Electric potential $\phi$ can then be expressed as

$$
\phi=\sum_{j=1}^{n} \phi_{j} N_{j}
$$


where $\phi_{j}$ is the field variable value at the node, $N_{j}$ is the shape function of finite element method, and $n$ is the number of nodes in each element.

The weak form of Eq. (1) can be obtained using the standard Galerkin method and it is written as

$$
\int_{\Omega} N_{i}(-\nabla \cdot \varepsilon \nabla \phi-\rho) d \Omega=0 \quad\left(i=1,2, \cdots, N_{\text {node }}\right)
$$

where $N_{\text {node }}$ is the number of nodes in analysis. Adopting the divergence theorem and applying boundary conditions, it obtains

$$
\int_{\Omega} \varepsilon \nabla N_{i} \cdot \nabla \phi d \Omega=\int_{\Omega} N_{i} \rho d \Omega \quad\left(i=1,2, \cdots, N_{\text {node }}\right)
$$

Substituting Eq. (3) into Eq. (5), the discretized system equation can be expressed in the following matrix form

$$
\mathbf{K u}=\mathbf{f}
$$

where

$$
\begin{gathered}
K_{i j}=\int_{\Omega} \varepsilon \nabla N_{i} \cdot \nabla N_{j} d \Omega \quad\left(i, j=1,2, \cdots, N_{\text {node }}\right) \\
u_{j}=\phi_{j} \quad\left(j=1,2, \cdots, N_{\text {node }}\right) \\
f_{i}=\int_{\Omega} N_{i} \rho d \Omega \quad\left(i=1,2, \cdots, N_{\text {node }}\right)
\end{gathered}
$$

\subsubsection{Diffusion equation}

Galerkin weak form for the diffusion equation in Eq. (2) can be derived as following,

$$
\begin{gathered}
\int_{\Omega}\left(v \nabla \times \mathbf{N}_{i} \cdot \nabla \times \dot{\mathbf{A}}+v \nabla \cdot \mathbf{N}_{i} \nabla \cdot \dot{\mathbf{A}}+j \omega \sigma \mathbf{N}_{i} \cdot \dot{\mathbf{A}}+\sigma \mathbf{N}_{i} \cdot \nabla \dot{\phi}-\mathbf{N}_{i} \cdot \dot{\mathbf{J}}_{s}\right) d \Omega=\mathbf{0} \\
\int_{\Omega} \nabla \mathbf{N}_{i} \cdot(j \omega \sigma \dot{\mathbf{A}}+\sigma \nabla \dot{\phi}) d \Omega=0
\end{gathered}
$$

Variable $\dot{\varphi}$ is introduced satisfying $\dot{\phi}=j \omega \dot{\varphi}$, so the symmetric form is obtained as 


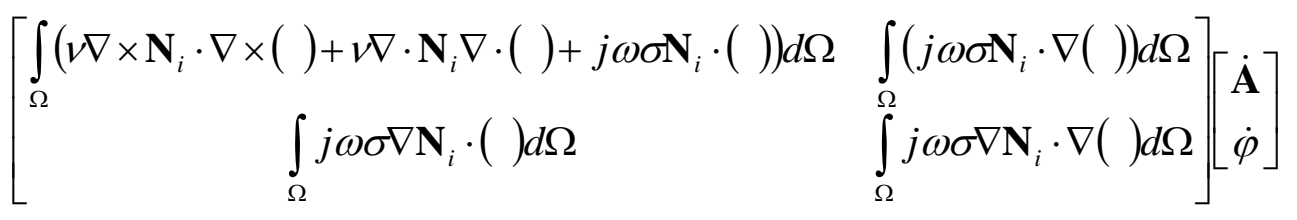

$$
\begin{aligned}
& =\left[\begin{array}{c}
\int_{\Omega}\left(\mathbf{N}_{i} \cdot \dot{\mathbf{J}}_{s}\right) d \Omega \\
0
\end{array}\right]
\end{aligned}
$$

Substituting the interpolated forms of $\dot{\mathbf{A}}$ and $\dot{\varphi}$, the matrix form is as following

$$
\left[\begin{array}{cccc}
\dot{\mathbf{S}}_{11} & \dot{\mathbf{S}}_{12} & \cdots & \dot{\mathbf{S}}_{1 N_{\text {node }}} \\
\dot{\mathbf{S}}_{21} & \dot{\mathbf{S}}_{22} & \cdots & \dot{\mathbf{S}}_{2 N_{\text {node }}} \\
\vdots & \vdots & \ddots & \vdots \\
\dot{\mathbf{S}}_{N_{\text {node }}} & \dot{\mathbf{S}}_{N_{\text {node }} 2} & \cdots & \dot{\mathbf{S}}_{N_{\text {nod }} N_{\text {node }}}
\end{array}\right]\left[\begin{array}{c}
\dot{\mathbf{U}}_{1} \\
\dot{\mathbf{U}}_{2} \\
\vdots \\
\dot{\mathbf{U}}_{N_{\text {node }}}
\end{array}\right]=\left[\begin{array}{c}
\dot{\mathbf{F}}_{1} \\
\dot{\mathbf{F}}_{2} \\
\vdots \\
\dot{\mathbf{F}}_{N_{\text {node }}}
\end{array}\right]
$$

where

$$
\begin{gathered}
\dot{\mathbf{S}}_{i j}=\left[\begin{array}{cccc}
\dot{S}_{i x, j x} & \dot{S}_{i x, j y} & \dot{S}_{i x, j z} & \dot{S}_{i x, j \varphi} \\
\dot{S}_{i y, j x} & \dot{S}_{i y, j y} & \dot{S}_{i y, j z} & \dot{S}_{i y, j \varphi} \\
\dot{S}_{i z, j x} & \dot{S}_{i z, j y} & \dot{S}_{i z, j z} & \dot{S}_{i z, j \varphi} \\
\dot{S}_{i \varphi, j x} & \dot{S}_{i \varphi, j y} & \dot{S}_{i \varphi, j z} & \dot{S}_{i \varphi, j \varphi}
\end{array}\right] \\
\dot{\mathbf{U}}_{j}=\left[\begin{array}{llll}
\dot{A}_{j x} & \dot{A}_{j y} & \dot{A}_{j z} & \dot{\varphi}_{j}
\end{array}\right]^{\mathrm{T}} \\
\dot{\mathbf{F}}_{i}=\left[\begin{array}{llll}
\dot{F}_{i x} & \dot{F}_{i y} & \dot{F}_{i z} & 0
\end{array}\right]^{\mathrm{T}}
\end{gathered}
$$

and all these items, $\dot{S}_{i \alpha, j \beta}, \dot{A}_{j \gamma}, \dot{F}_{i \gamma}, \dot{\varphi}_{j}(\alpha, \beta=x, y, z, \varphi ; \gamma=x, y, z)$, are expressed as integral equations of $N_{i}, N_{j}, \partial N_{i} / \partial r, \partial N_{j} / \partial r(r=x, y, z)$ terms.

\subsection{Node-based smoothed finite element method (NS-FEM)}

To carry out the integration in Eqs. (7), (9), (13) and (15), based on the background mesh, the integration domain $\Omega$ is further divided into $N_{\text {node }}$ non-overlapping smoothing domains of polyhedron (polygon for 2D) $\Omega_{k}^{s}\left(k=1,2, \ldots, N_{\text {node }}\right)$ centered by node $k$. Fig. 1 is the schematic of a typical nodal smoothing domain in $2 \mathrm{D}$ and $3 \mathrm{D}$ domain. The boundary of the smoothing domain $\Omega_{k}^{s}$ is labeled as $\Gamma_{k}^{s}$ and the union of all $\Omega_{k}^{s}$ forms exactly the global domain $\Omega$. 
The supporting nodes $\mathbf{n}_{k}^{s}$ for node $k$ are formed by all its adjacent nodes including itself, and the number of supporting nodes is defined as $N_{k}^{s}$.

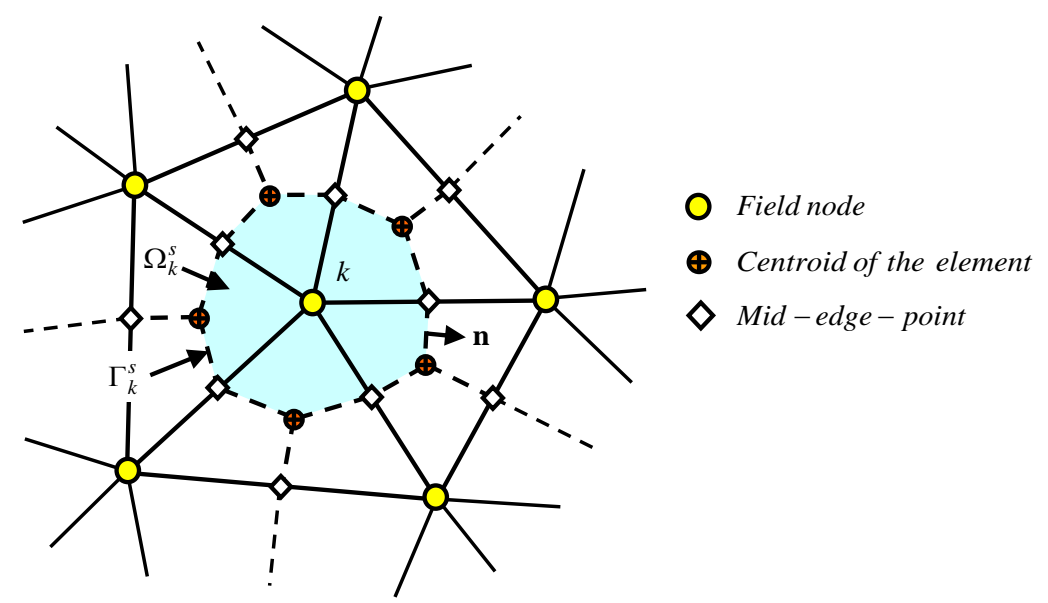

(a)

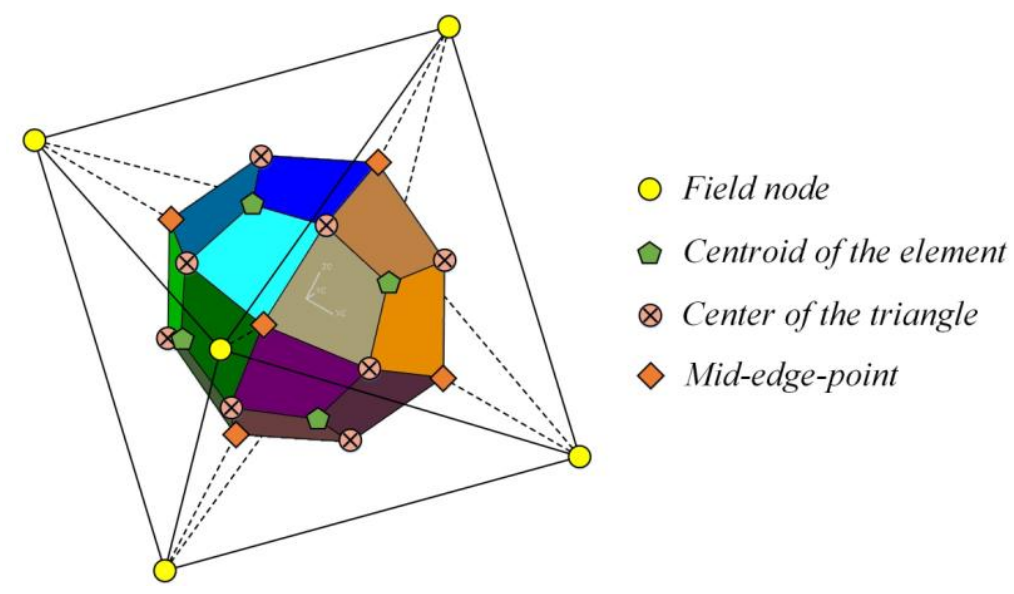

(b)

Fig. 1. The schematic of a node-based smoothing domain for node $k$ : (a) 2D smoothing domain; (b) 3D smoothing domain.

Different from FEM theory, the integration shown in Eqs. (7), (9), (13) and (15) can be performed upon each smoothing domain $\Omega_{k}^{s}$ as in Fig. 1 when adopting the node-based scheme. And then items $\partial N_{i} / \partial r, \partial N_{j} / \partial r(r=x, y, z)$ are smoothed on $\Omega_{k}^{s}$, and are finally computed upon the boundary $\Gamma_{k}^{s}$ according to the divergence theorem. This is the main idea of node-based smoothed finite element method (NS-FEM) proposed by G.R. Liu and detailed derivations can be seen in [8]. 


\subsection{Stable nodal integration method (SNIM)}

NS-FEM fails to ensure temporal stability in mechanical problems, which can be reflected by the non-zero energy modes produced by NS-FEM [25, 31]. It is generally believed that NS-FEM provides "overly-soft" system, while traditional FEM usually offers stable results for various problems, which makes it the most commonly used method adopted by commercial softwares. Therefore, the authors present a stable nodal integration method (SNIM) in consideration of the integration scheme of FEM.

By FEM theory, constant values of $\partial N_{i} / \partial r, \partial N_{j} / \partial r(r=x, y, z)$ are obtained if linear interpolation is adopted in triangular or tetrahedral elements, and one integration point at the geometric centroid of each element is chosen to calculate the stiffness matrix. Domain $\Omega_{k}^{s}$ around node $k$ and the integration points in FEM formulation $g_{i}^{e}\left(i=1, \cdots, N_{e}^{s}\right)$ is sketched in Fig. 2. $N_{e}^{s}$ is the number of related elements around node $k$. To obtain the stable nodal integration scheme, domain $\Omega_{k}^{s}$ is approximated as a sphere (or circular) domain $\Omega_{k}^{s c}$ with the same volume (or area) $A_{k}^{s}$, which is then subdivided into six (or four) sub-domains, and the chosen integration points $g_{i}^{n}(i=1,2,3,4,5,6)$ lie in $\mathrm{x}$-axis, $\mathrm{y}$-axis and $\mathrm{z}$-axis and keep the same distance $l_{c}$ to node $k$, as layout in Fig. 2. $l_{c}$ is the radius of domain $\Omega_{k}^{s c}$, which can be calculated as following.

$$
\begin{array}{cc}
l_{c}=\sqrt{A_{k}^{s} / \pi} & \text { for } 2 D \\
l_{c}=\sqrt[3]{3 A_{k}^{s} / 4 \pi} & \text { for } 3 D
\end{array}
$$




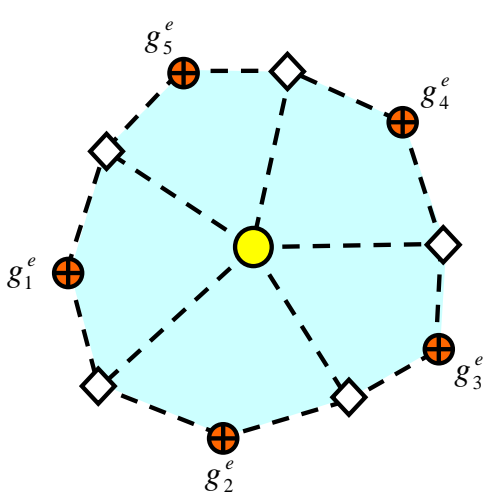

(a)

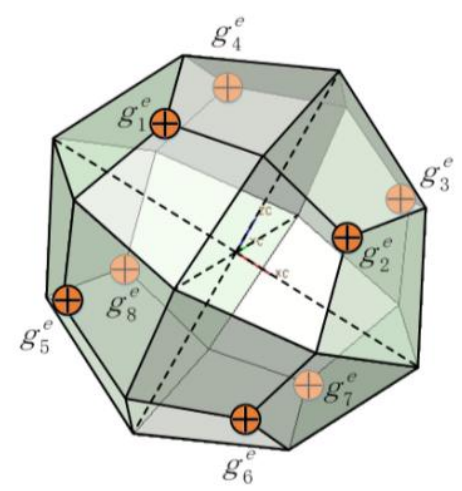

(c)

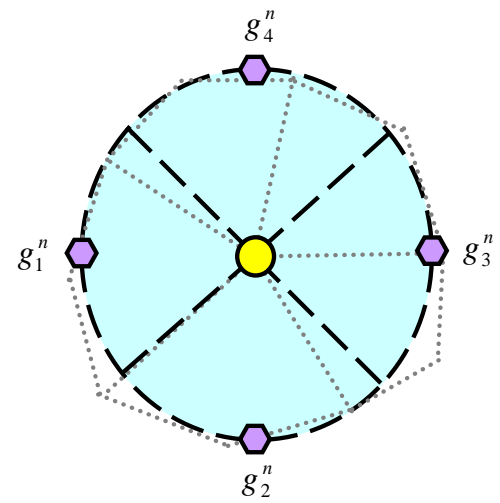

(b)

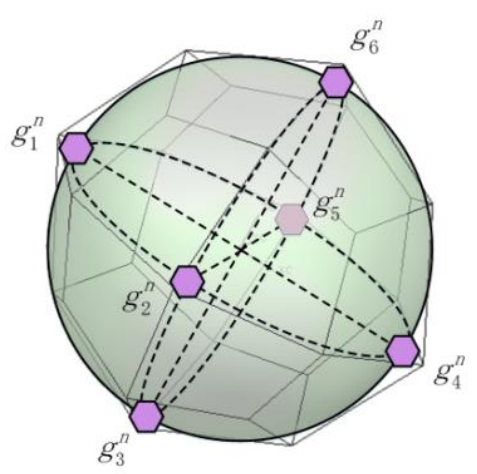

(d)

Fig. 2. The integration domain and integration points of FEM and SNIM: (a) FEM for 2D; (b) SNIM for 2D; (c) FEM for 3D; (d) SNIM for 3D.

The reason for such operation is not only because it is easy to implement, but also that the discrepancy between FEM and NS-FEM is introduced into SNIM to some extent.

Assuming $\partial N_{i} / \partial r, \partial N_{j} / \partial r(r=x, y, z)$, named as $D N$, in $\Omega_{k}^{s c}$ is continuous and derivable at the first order, the Taylor expansion is

$$
D N=D N_{k}+\frac{\partial D N}{\partial x}\left(x-x_{k}\right)+\frac{\partial D N}{\partial y}\left(y-y_{k}\right)+\frac{\partial D N}{\partial z}\left(z-z_{k}\right)
$$

So $D N$ of the six sub-domains are $D N_{1}^{s c}, D N_{2}^{s c}, D N_{3}^{s c}, D N_{4}^{s c}, D N_{5}^{s c}$ and $D N_{6}^{s c}$, which can be expressed as

$$
\begin{aligned}
& D N_{1}^{s c}=D N_{k}-\frac{\partial D N}{\partial x} \cdot l_{c}, D N_{2}^{s c}=D N_{k}-\frac{\partial D N}{\partial y} \cdot l_{c}, D N_{3}^{s c}=D N_{k}-\frac{\partial D N}{\partial z} \cdot l_{c} \\
& D N_{4}^{s c}=D N_{k}+\frac{\partial D N}{\partial x} \cdot l_{c}, D N_{5}^{s c}=D N_{k}+\frac{\partial D N}{\partial y} \cdot l_{c}, D N_{6}^{s c}=D N_{k}+\frac{\partial D N}{\partial z} \cdot l_{c}
\end{aligned}
$$


So terms of the stiffness matrix can be calculated on six sub-domains, for instance

$$
\int_{\Omega_{k}^{s c}} \frac{\partial N_{i}}{\partial r} \cdot \frac{\partial N_{j}}{\partial t} d \Omega_{k}^{s c}=\sum_{m=1}^{6}\left(\left(\frac{\partial N_{i}}{\partial r}\right)_{m} \cdot\left(\frac{\partial N_{j}}{\partial t}\right)_{m}\right) \cdot \frac{A_{k}^{s}}{6} \quad(r, t=x, y, z)
$$

Substituting Eq. (18) into Eq. (19), it turns out to be

$$
\begin{aligned}
\int_{\Omega_{k}^{s c}} \frac{\partial N_{i}}{\partial r} \cdot \frac{\partial N_{j}}{\partial t} d \Omega_{k}^{s c} & =(D N r \cdot D N t)_{k} \cdot A_{k}^{s}+\Delta D N r_{x} \cdot \Delta D N t_{x} \cdot \frac{A_{k}^{s}}{3} \\
& +\Delta D N r_{y} \cdot \Delta D N t_{y} \cdot \frac{A_{k}^{s}}{3}+\Delta D N r_{z} \cdot \Delta D N t_{z} \cdot \frac{A_{k}^{s}}{3}
\end{aligned}
$$

where $D N r=\partial N_{i} / \partial r, D N t=\partial N_{j} / \partial t(r, t=x, y, z)$, and

$$
\begin{aligned}
& \Delta D N r_{x}=\frac{\partial D N r}{\partial x} \cdot l_{c}, \Delta D N r_{y}=\frac{\partial D N r}{\partial y} \cdot l_{c}, \Delta D N r_{z}=\frac{\partial D N r}{\partial z} \cdot l_{c} \\
& \Delta D N t_{x}=\frac{\partial D N t}{\partial x} \cdot l_{c}, \Delta D N t_{y}=\frac{\partial D N t}{\partial y} \cdot l_{c}, \Delta D N t_{z}=\frac{\partial D N t}{\partial z} \cdot l_{c}
\end{aligned}
$$

In the same way, all items in Eqs. (7), (9), (13) and (15) can be obtained by SNIM, and are calculated on the boundary by the divergence theorem.

Finally, it is worth mentioning that the six (or four) integration points are just temporary variables which are accomplished equivalently by one point integration and the stabilization terms, and this can be realized by the addition of several lines on original code. And the efficacy of these terms can be witnessed by the following examples. By the way, integration points of SNIM are nodes from the mesh. Therefore, all information, such as eddy current density or magnetic flux density, are carried on nodes, so they can be easily obtained and transferred without complicated post processing. In general, the proposed SNIM formulation can be considered as a kind of particle method, except that background mesh is needed in the early stage of computation. 


\section{Applications and results}

In this section, examples for Poisson equation and diffusion equation of $2 \mathrm{D}$ and 3D geometries are presented using FEM, NS-FEM and SNIM to perceive performance of each method. The error norms and convergence rate are defined as

$$
\begin{gathered}
L^{2} \operatorname{err}(h)=\left\{\int_{\Omega}\left(\mathbf{u}^{\text {num }}-\mathbf{u}^{\text {exact }}\right)^{2} d \Omega\right\}^{\frac{1}{2}} \\
H^{1} \operatorname{err}(h)=\left\{\int_{\Omega}\left[\left(\mathbf{u}^{\text {num }}-\mathbf{u}^{\text {exact }}\right)^{2}+\sum_{\alpha=x, y, z}\left(\mathbf{u}_{\alpha}^{\text {num }}-\mathbf{u}_{\alpha}^{\text {exact }}\right)^{2}\right] d \Omega\right\}^{\frac{1}{2}} \\
r=\log _{2}\left(\frac{\operatorname{err}(h)}{\operatorname{err}(2 h)}\right)
\end{gathered}
$$

where $\mathbf{u}^{\text {num }}$ is the potential obtained by numerical methods, $\mathbf{u}^{\text {exact }}$ is the analytical potential value, $\mathbf{u}_{x}, \mathbf{u}_{y}$ and $\mathbf{u}_{z}$ are the derivatives of $\mathbf{u}$ with respect to $x, y$ and $z$ respectively, and $h$ is the equivalent length between nodes. When calculating $L^{2}$ and $H^{1}$ error norms for Poisson equation, parameter $\mathbf{u}$ in Eqs. (22) and (23) should be written into scalar form $u$, and the specific expressions are omitted for simple.

\subsection{D Poisson equation}

Consider the following Poisson equation

$$
\nabla^{2} u(x, y)=-8 \pi^{2} \sin (2 \pi x) \sin (2 \pi y),(x, y) \in[-0.5,0.5] \times[-0.5,0.5]
$$

with boundary condition

$$
u(x, y)=\sin (2 \pi x) \sin (2 \pi y)
$$

The analytical solution for this problem is as demonstrated in Eq. (26).

Figs. 3 and 4 show the convergence of the solutions in $L_{2}$ error norm and $H_{1}$ error norm. It can be found that the present method is more accurate than FEM in $H_{1}$ 
error norm. And the convergence rate of SNIM is higher than that of FEM in both $L_{2}$ and $H_{1}$ norms, which varies from 1.95 to 2.05 in $L_{2}$ norm, and varies from 0.74 to 1.56 in $H_{1}$ norm.

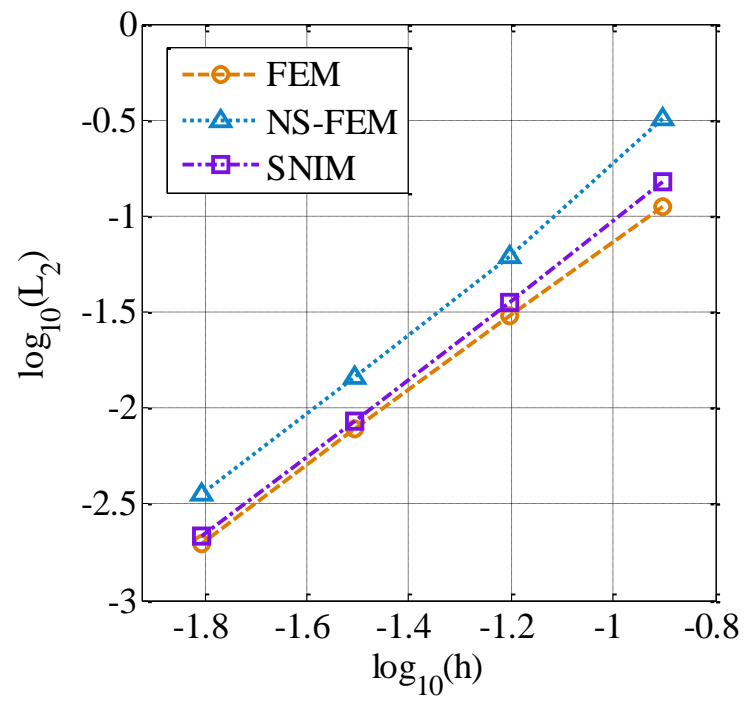

Fig. 3. Error estimation in $L_{2}$ norm for 2D Poisson equation.

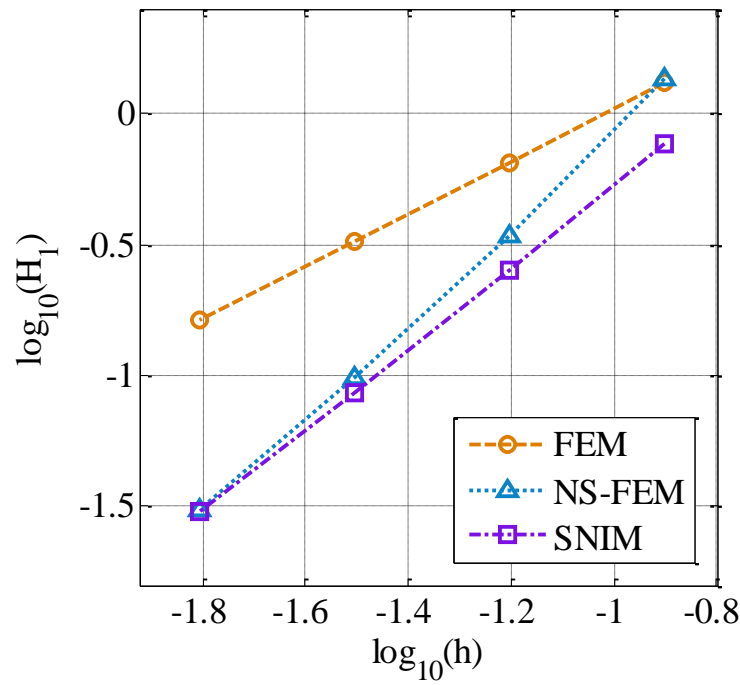

Fig. 4. Error estimation in $H_{1}$ norm for 2D Poisson equation.

To further study features of the proposed solution, derivative results of the potential function are plotted in Figs. 5 and 6. It can be observed that results obtained by SNIM are more accurate than ones obtained by traditional FEM and NS-FEM. Since the gradient result is quite crucial in electromagnetic analysis, for the magnetic flux density is obtained by $\mathbf{B}=\nabla \times \mathbf{A}$, SNIM acquires its superiority in this respect. 


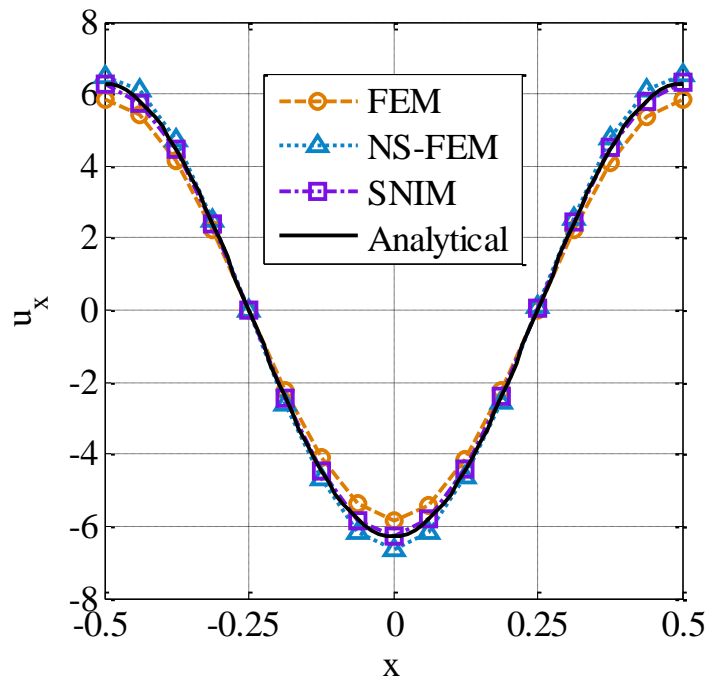

Fig. 5. Results of $\partial u / \partial x$ on line $y=-l y / 4$ for 2D Poisson equation.

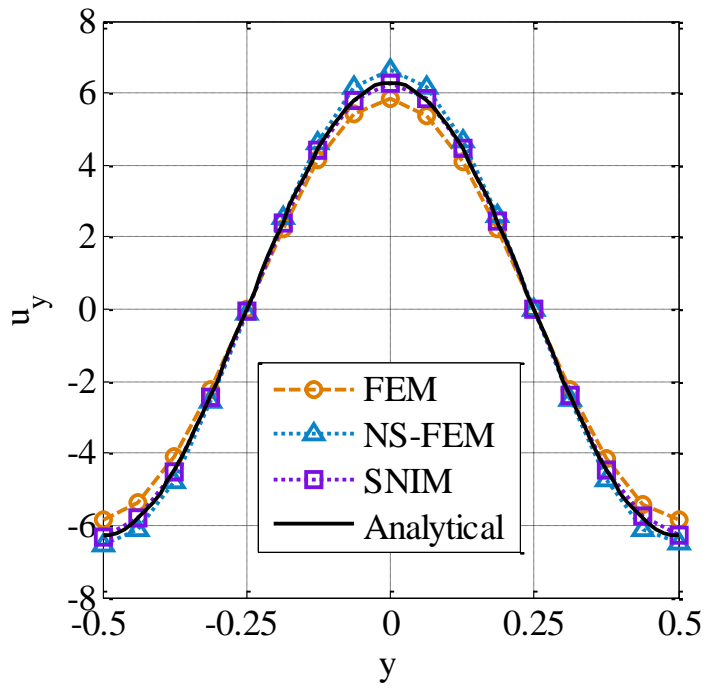

Fig. 6. Results of $\partial u / \partial y$ on line $x=+l x / 4$ for $2 \mathrm{D}$ Poisson equation.

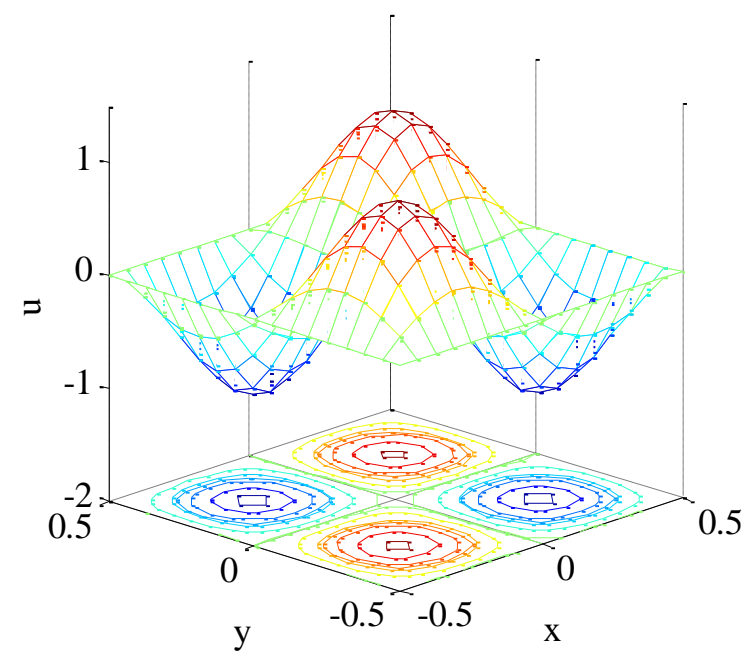

Fig. 7. Surface plot of SNIM and the analytical solution for 2D Poisson equation. 
And then, a typical surface plot of SNIM and the analytical solution by $17 \times 17$ nodes mesh is shown in Fig. 7, which proves the good agreement of SNIM result with the analytical one. Finally, error norm of $H_{1}$ with respect to CPU time is plotted in Fig. 8, which testifies the superior efficiency of SNIM.

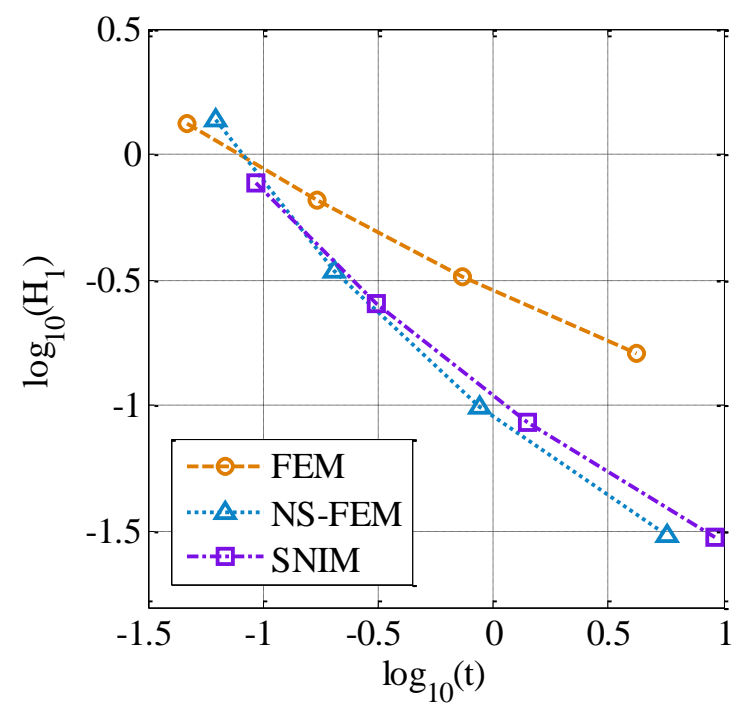

Fig. 8. $H_{1}$ error norm with respect to CPU time for 2D Poisson equation.

\subsection{D eddy current problem}

The eddy current analysis of a unit circular disk is investigated. The Dirichelet boundary conditions are imposed as

$$
\mathbf{A}(x, y)=\left(\begin{array}{c}
-y \\
x
\end{array}\right), V(x, y)=0
$$

The analytical solution for this problem is

$$
\mathbf{A}(x, y)=\frac{I_{1}\left(j^{\frac{1}{2}}(\omega \sigma \mu)^{\frac{1}{2}} r\right)}{r I_{1}\left(j^{\frac{1}{2}}(\omega \sigma \mu)^{\frac{1}{2}}\right)}\left(\begin{array}{c}
-y \\
x
\end{array}\right), V(x, y)=0
$$

where $I_{1}$ is the modified Bessel function of the first kind. $\omega=1, \sigma=1$ and $\mu=1$ are adopted as the material properties, so the analytical result is as shown in Fig. 9. 


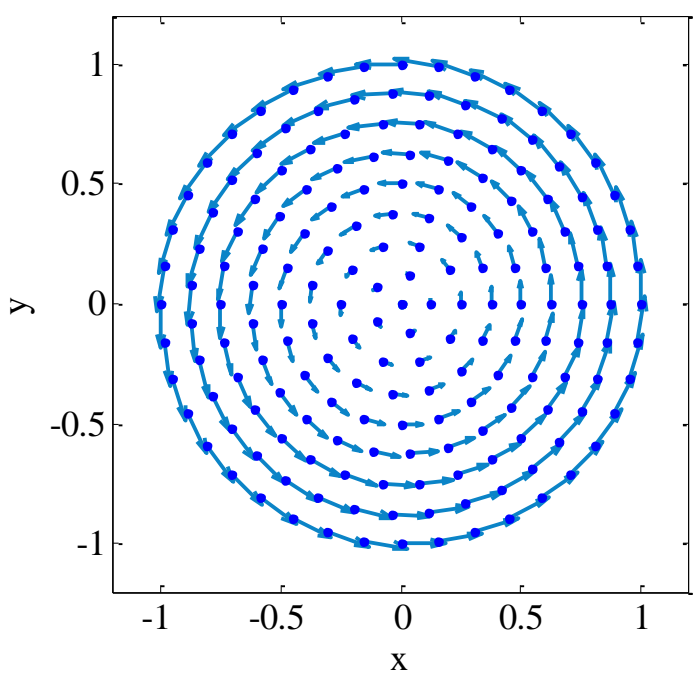

Fig. 9. Analytical result for circular disk.

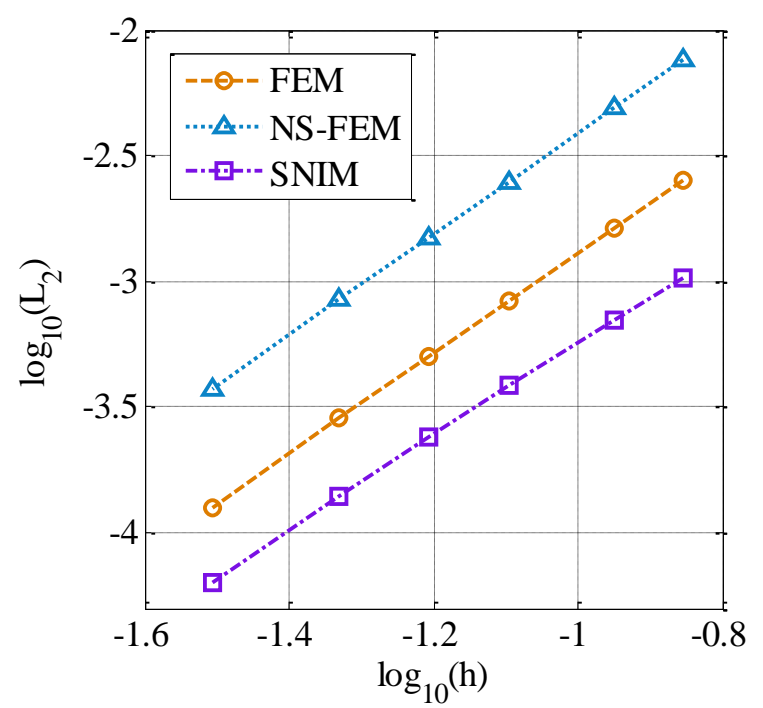

Fig. 10. Error estimation in $L_{2}$ norm for circular disk.

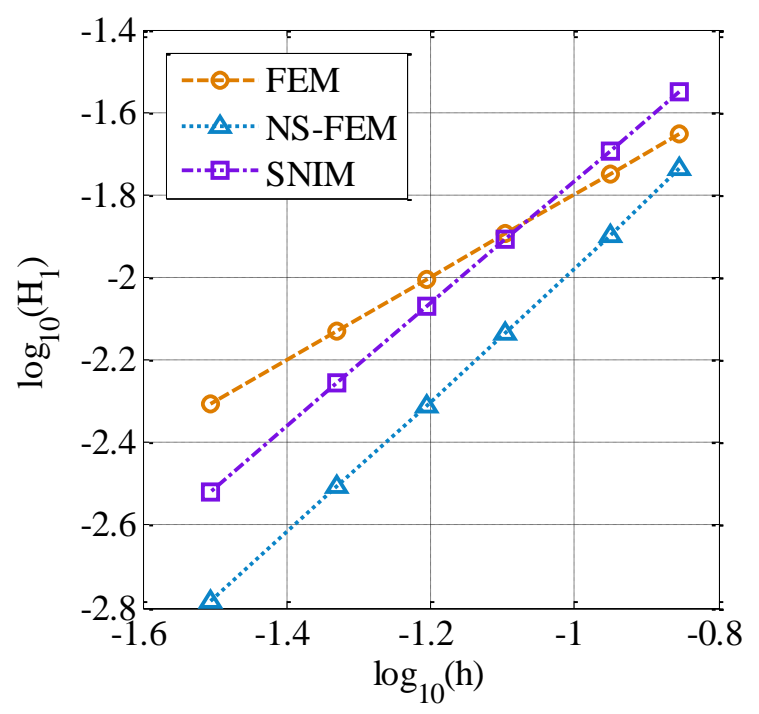

Fig. 11. Error estimation in $H_{1}$ norm for circular disk. 
The error norms in $L_{2}$ and $H_{1}$ of the numerical results are calculated and plotted in Figs. 10 and 11. It can be found that SNIM solution is more accurate in $L_{2}$ error norm and maintains higher convergence rate than FEM in $H_{1}$ error norm, which varies from 1.00 to 1.48 .

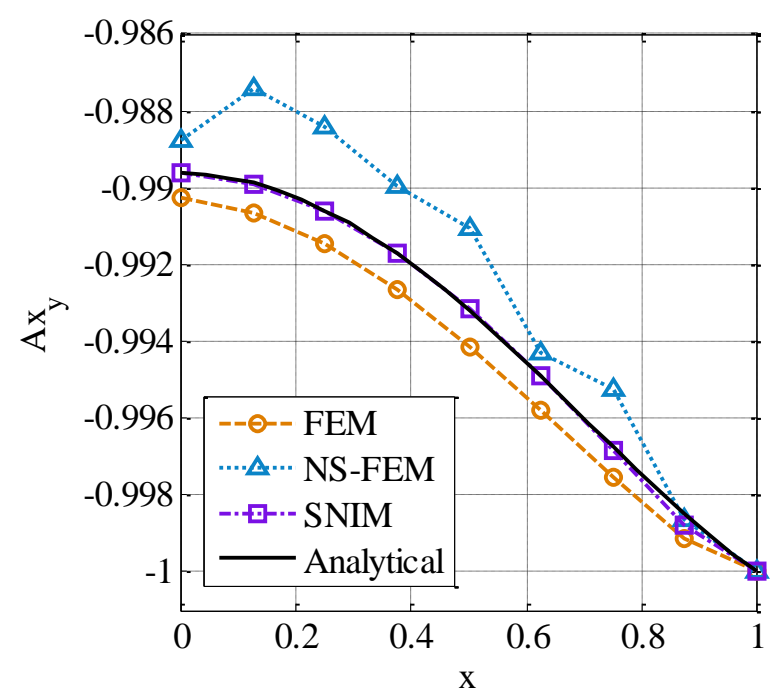

Fig. 12. Results of $\partial A x / \partial y$ along $x$-axis for circular disk.

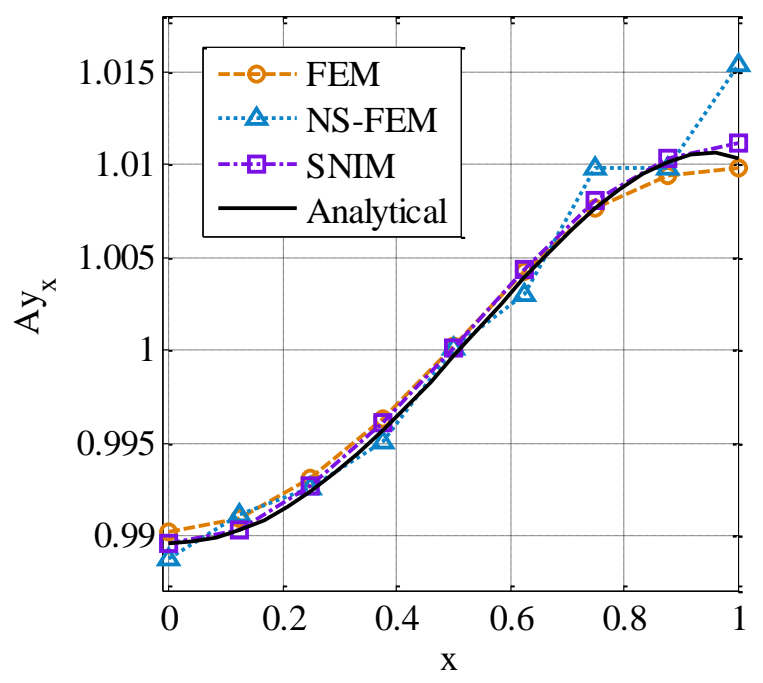

Fig. 13. Results of $\partial A y / \partial x$ along $x$-axis for circular disk.

The resulted derivatives along $\mathrm{x}$-axis are plotted in Figs. 12 and 13, which testify that results of SNIM are fairly in keeping with reference results even if a relatively coarse mesh is employed. And also, it can be witnessed that NS-FEM offers fluctuant 
results even for this quasi-static problem. Therefore, the instability of NS-FEM can not be confined into time dependent situations in electromagnetics, so the development of stable integration scheme in this work seems to be quite necessary.

\subsection{D Poisson equation}

A 3D Poisson equation is considered in this subsection as

$$
\nabla^{2} u(x, y, z)=-12 \pi^{2} \sin (2 \pi x) \sin (2 \pi y) \sin (2 \pi z)
$$

where $(x, y, z) \in[-0.5,0.5] \times[-0.5,0.5] \times[-0.5,0.5]$, with boundary condition

$$
u(x, y, z)=\sin (2 \pi x) \sin (2 \pi y) \sin (2 \pi z)
$$

The analytical solution for this problem can also be expressed as Eq. (30).

Again for this problem, $L_{2}$ and $H_{1}$ error norms are shown in Figs. 14 and 15. In $L_{2}$ error norm, the proposed solution fails to defeat FEM in accuracy, while it gains a bit advantage in convergence rate. However, in $H_{1}$ error norm, SNIM gets a higher convergence rate of 1.41 than FEM of 1.01 while providing more accurate results.

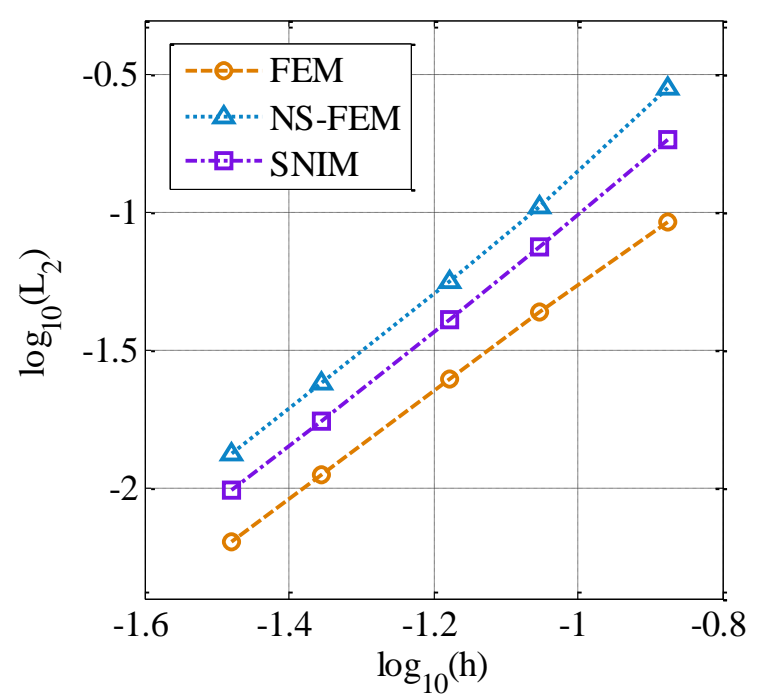

Fig. 14. Error estimation in $L_{2}$ norm for 3D Poisson equation. 


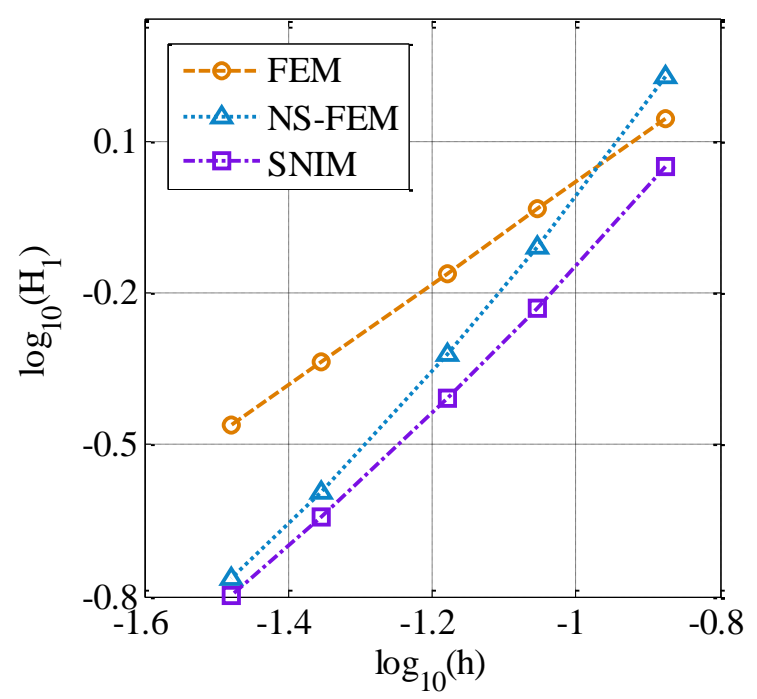

Fig. 15. Error estimation in $H_{1}$ norm for 3D Poisson equation.

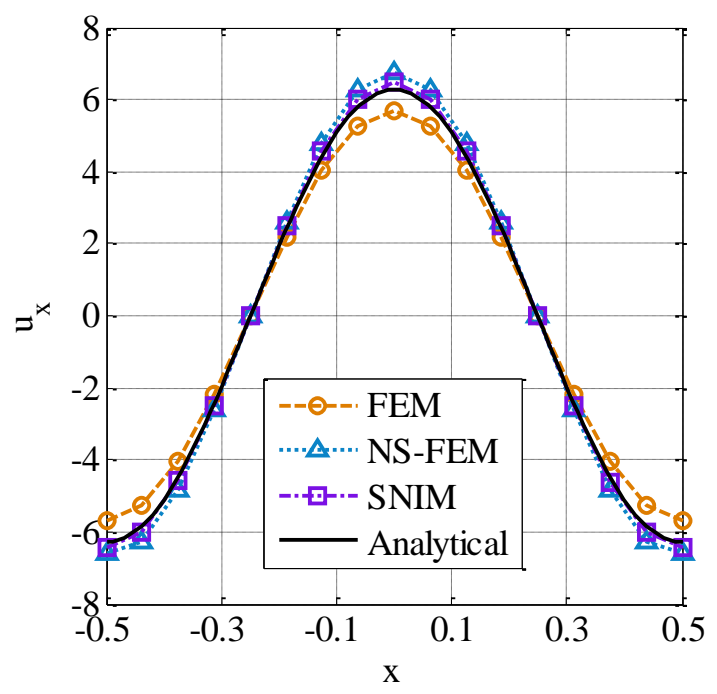

Fig. 16. Results of $\partial u / \partial x$ on line $y=-l y / 4, z=-l z / 4$ for 3D Poisson equation.

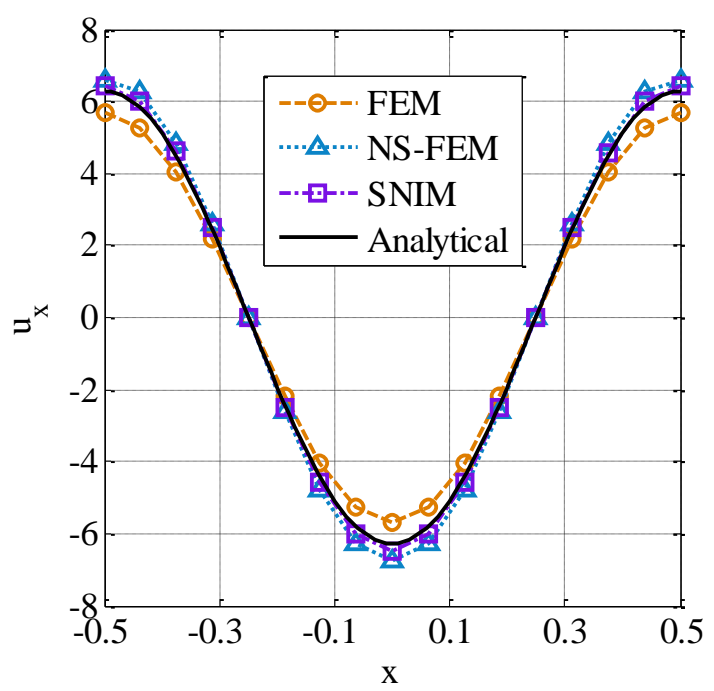

Fig. 17. Results of $\partial u / \partial x$ on line $y=+l y / 4, z=-l z / 4$ for 3D Poisson equation. 
And also, derivative values of the field variable are obtained on two lines in this case, as shown in Figs. 16 and 17. It observes that the presented method completes well in this $3 \mathrm{D}$ benchmark problem.

\subsection{D eddy current problem}

To illustrate the application of the proposed method to 3D eddy current problems, the developed codes are used to solve Problem 7 of TEAM (Testing Electromagnetic Analysis Methods) workshop benchmark problems in this subsection. The computation results are compared with results obtained by experiment, which were presented at the workshop [42-44]. The computation model is consist of an asymmetrical conductor with a hole, an exciting coil, and the surrounding air, as shown in Fig. 18. The conducting plate has a conductivity of $3.526 \times 10^{7} \mathrm{~S} / \mathrm{m}$, and the coil is excited with 2472 Ampere-Turns. Computation results in which the frequency of exciting coil is $200 \mathrm{~Hz}$ are calculated and compared with experimental results. The eddy current density is simulated along the lines A3-B3 on the upper surface of the conductor, and A4-B4 on the bottom surface of the conductor. The magnetic flux density is simulated along the lines A1-B1 $(\mathrm{y}=72 \mathrm{~mm})$ and A2-B2 $(y=144 \mathrm{~mm})$ at the middle of the exciting coil and the conductor.

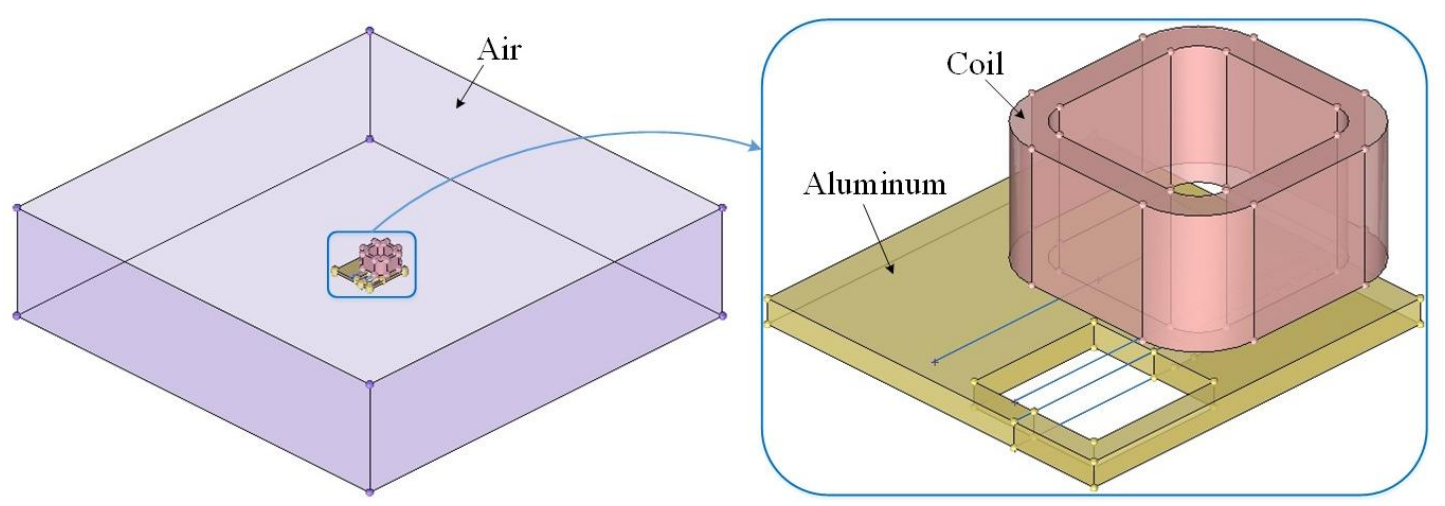

Fig. 18. Computation model of TEAM Problem 7. 


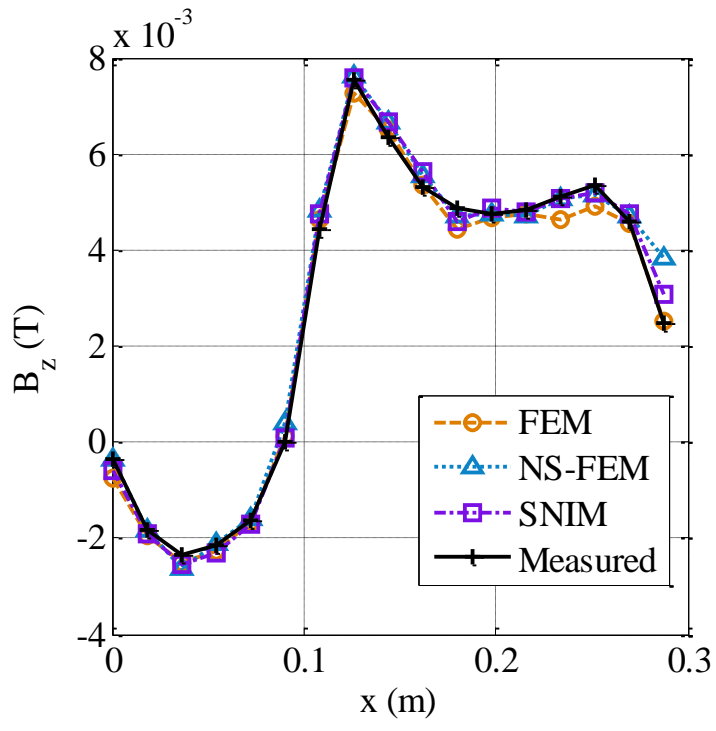

(a)

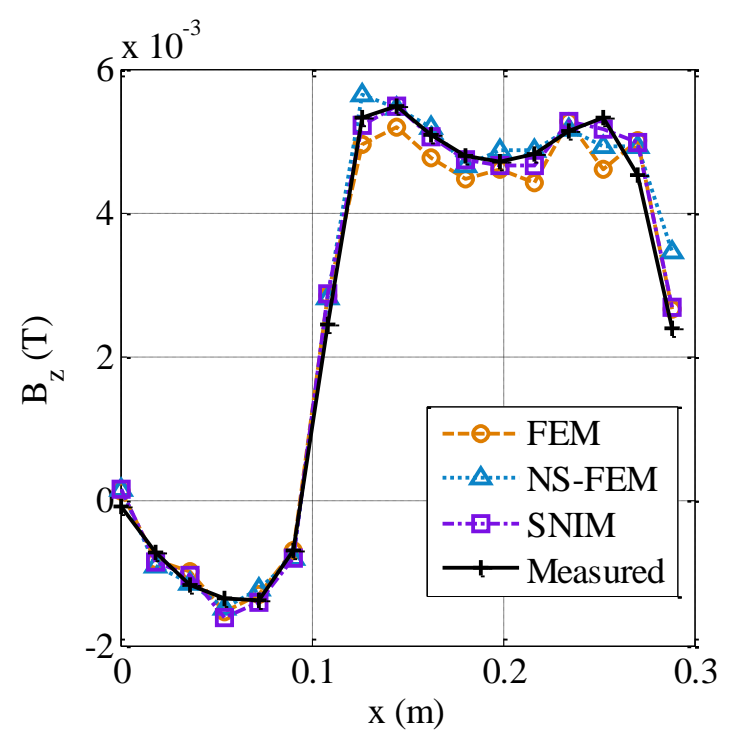

(b)

Fig. 19. Magnetic flux density $B_{\mathrm{z}}$ along certain lines for TEAM Problem 7 by FEM, NS-FEM and SNIM solutions: (a) line A1-B1; (b) line A2-B2.

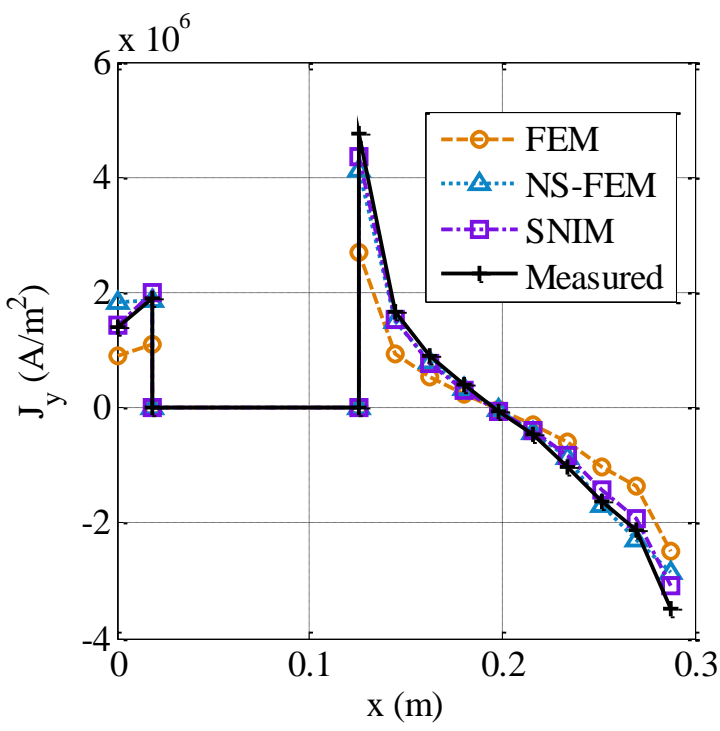

(a)

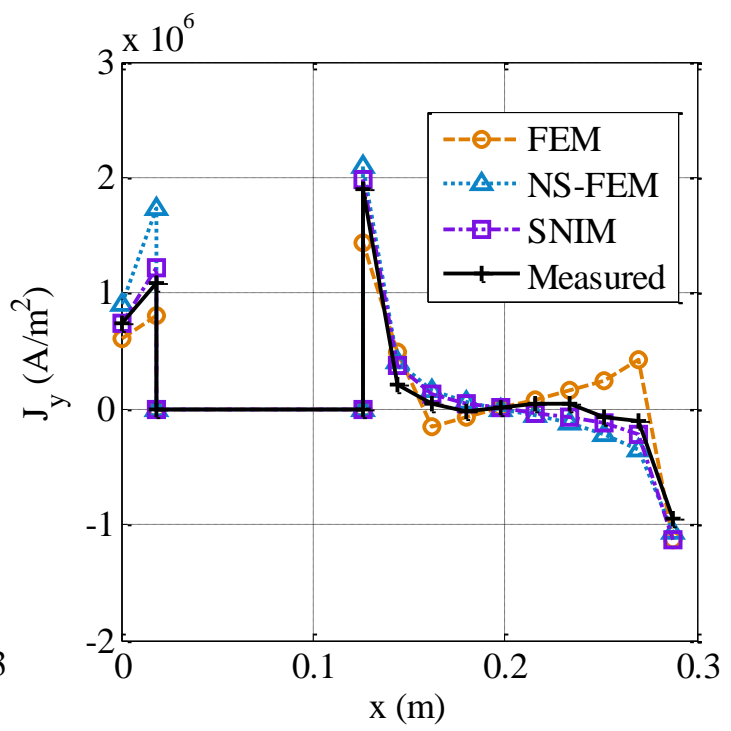

(b)

Fig. 20. Eddy current density $J_{\mathrm{y}}$ along certain lines for TEAM Problem 7 by FEM, NS-FEM and SNIM solutions: (a) line A3-B3; (d) line A4-B4.

Figs. 19 and 20 show the comparisons between computational and experimental results by FEM, NS-FEM and SNIM solutions. It is visible that SNIM, which generally acquires quite favorable results in keeping with experimental ones, surpass traditional FEM and NS-FEM in accuracy. Furthermore, performance of the proposed solution is studied by comparison with other approaches through this case. Since the 
edge-based smoothed finite element method (ES-FEM) has been proved to be very effective in solving various problems [12, 34], a comparison with ES-FEM is executed and the obtained results are as shown in Figs. 21 and 22. It can be seen that ES-FEM performs relatively poor in solving this quasi-static electromagnetic problem and super accuracy is acquired by SNIM solution.

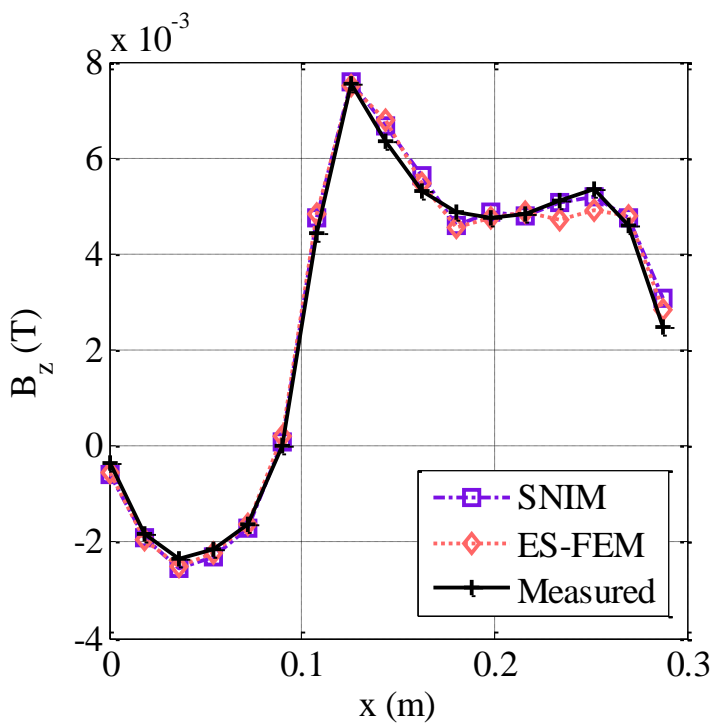

(a)

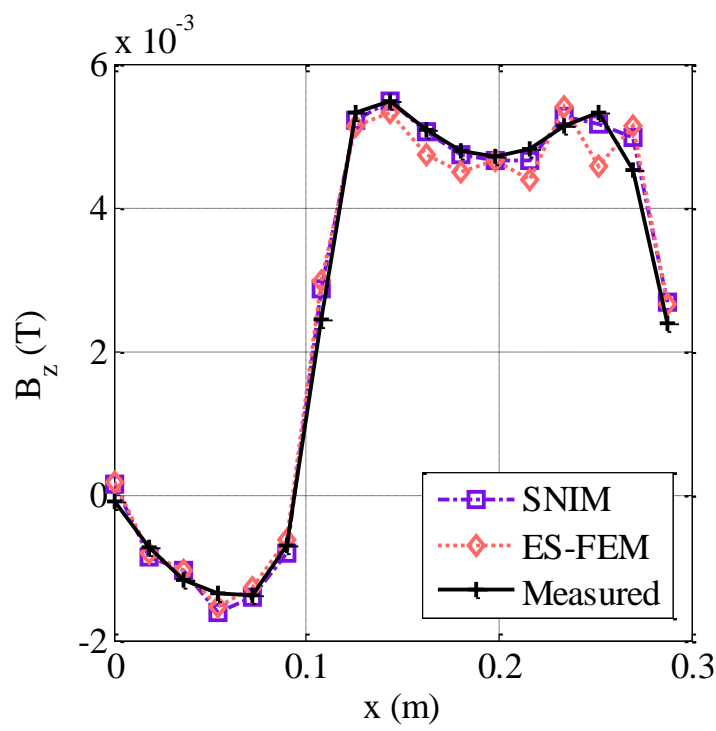

(b)

Fig. 21. Magnetic flux density $B_{z}$ along certain lines for TEAM Problem 7 by SNIM and ES-FEM solutions: (a) line A1-B1; (b) line A2-B2.

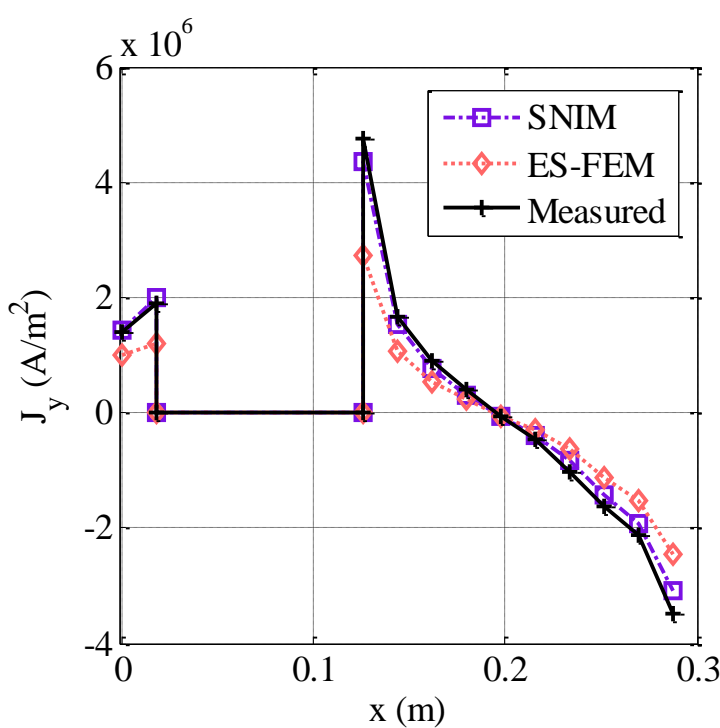

(a)

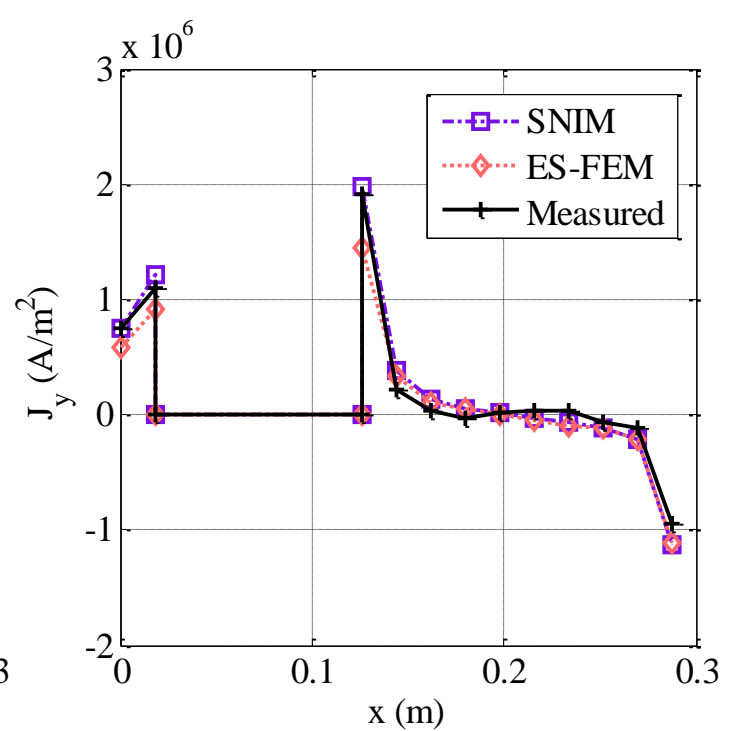

(b)

Fig. 22. Eddy current density $J_{\mathrm{y}}$ along certain lines for TEAM Problem 7 by SNIM and ES-FEM solutions: (a) line A3-B3; (d) line A4-B4. 
Except for accuracy, the computational cost analysis is also necessary to assess one new developed algorithm [45]. The results in this subsection have all been obtained under the same platform in a computer with CPU Inter Core i5-6600 at 3.30GHz with 4.00GB of RAM memory and the Microsoft Windows 7 Professional X64 Edition V2009 operating system. All codes have been written in Intel Visual Fortran and run with single thread. The times measured are CPU times including the assembly of system matrix and solving of system equations. Table 1 shows the iteration steps of the ICCG method (incomplete Cholesky decomposition conjugate gradient method), the control errors, and computation times of the adopted solutions. It can be seen that the computation time of SNIM is longer than that of FEM, for SNIM gets wider bandwidth through the smoothing operation. The cost of SNIM and ES-FEM are nearly the same, so the attractive feature of SNIM can be proved. And also, it's worth pointing out that the proposed method uses much less iteration steps than FEM, which may win the favor for large-scale problems or in nonlinear analysis, and a preconditioning method suitable for SNIM will make it more competitive. Finally, we would like to indicate that the main purpose of the proposed formulation is the enlargement of solution types, since the nodal FEM adopting linear triangular element and tetrahedral element would usually bring out relatively poor results and are not commonly used for the numerical computation of electromagnetic problems.

Table 1 Computation parameters of various solutions.

\begin{tabular}{cccc}
\hline Solution & Iterations of ICCG & Control error & CPU time \\
\hline FEM & 468 & $6.610 \mathrm{E}-8$ & 112.664 \\
NS-FEM & 582 & $8.406 \mathrm{E}-8$ & 324.544 \\
SNIM & 284 & $1.870 \mathrm{E}-7$ & 258.962 \\
ES-FEM & 316 & $1.860 \mathrm{E}-7$ & 255.935 \\
\hline
\end{tabular}




\section{Conclusion}

In this work, based on linear triangular (for 2D) and tetrahedral (for 3D) background mesh, SNIM solution is formulated for electromagnetic problems in static and quasi-static analysis. Characteristics of SNIM can be summarized into four aspects. Firstly, the proposed method completes well in above problems, and the accuracy improves by SNIM. Secondly, higher convergence rate can be obtained by SNIM for $H_{1}$ error norm, and derivatives can be captured with high accuracy. Thirdly, although SNIM occupies more computation time than FEM for its wider bandwidth, less iteration steps can still be obtained. So a preconditioning method suitable for SNIM will make it more competitive. Fourthly, since data are carried on nodes by SNIM, so it can be coupled with other methods (even with meshless methods) conveniently and applications on coupled-field analysis are to be expected since it has been explored and proved to be effective in various fields. All in all, numerical examples show the adequacy of the proposed methodology for electromagnetic analysis, and its attractive features produce potential for further study.

\section{Acknowledgments}

The support of National Science Foundation of China (11472101), State Key Program of National Natural Science of China (61232014) and Hunan Provincial Natural Science Foundation of China (2015JJ3037) are gratefully acknowledged.

\section{Conflict of Interest}

The authors declare that they have no conflict of interest.

\section{References}

1. T. Belytschko, Y.Y. Lu, L. Gu, Element-free Galerkin methods, Int. J. Numer. 
Methods Eng. 37 (1994) 229-256.

2. V. Čingoski, N. Miyamoto, H. Yamashita, Element-free Galerkin method for electromagnetic field computations, IEEE Trans. Magn. 34 (5) (1988) 3236-3239.

3. G.F. Parreira, E.J. Silva, A.R. Fonseca, R.C. Mesquita, The element-free Galerkin method in three-dimensional electromagnetic problems, IEEE Trans. Magn. 42 (4) (2006) 711-714.

4. S.J. Lai, B.Z. Wang, Y. Duan, Meshless radial basis function method for transient electromagnetic computations, IEEE Trans. Magn. 44 (10) (2008) 2288-2295.

5. S.A. Viana, R.C. Mesquita, Moving least square reproducing kernel method for electromagnetic field computation, IEEE Trans. Magn. 35 (3) (1999) 1372-1375.

6. G.R. Liu, A G space theory and a weakened weak (W2) form for a unified formulation of compatible and incompatible methods: Part I theory, Int. J. Numer. Methods Eng. 81 (9) (2010) 1093-1126.

7. G.R. Liu, A G space theory and a weakened weak $\left(\mathrm{W}^{2}\right)$ form for a unified formulation of compatible and incompatible methods: Part II applications to solid mechanics problems, Int. J. Numer. Methods Eng. 81 (9) (2010) 1127-1156.

8. G.R. Liu, T. Nguyen-Thoi, H. Nguyen-Xuan, K.Y. Lam, A node-based smoothed finite element method (NS-FEM) for upper bound solutions to solid mechanics problems, Comput. Struct. 87 (2009) 14-26.

9. T. Nguyen-Thoi, H.C. Vu-Do, T. Rabczuk, H. Nguyen-Xuan, A node-based smoothed finite element method (NS-FEM) for upper bound solution to visco-elastoplastic analyses of solids using triangular and tetrahedral meshes, 
Comput. Methods Appl. Mech. Eng. 199 (2010) 3005-3027.

10. G.R. Liu, G.Y. Zhang, K.Y. Dai, Y.Y. Wang, Z.H. Zhong, G.Y. Li, X. Han, A linearly conforming point interpolation method (LC-PIM) for 2D solid mechanics problems, Int. J. Comput. Methods 2 (4) (2005) 645-665.

11. G.R. Liu, K.Y. Dai, T.T. Nguyen, A smoothed finite element method for mechanics problems, Comput. Mech. 39 (2007) 859-877.

12. H. Nguyen-Xuan, G.R. Liu, S. Bordas, S. Natarajan, T. Rabczuk, An adaptive singular ES-FEM for mechanics problems with singular field of arbitrary order, Comput. Methods Appl. Mech. Eng. 253 (2013) 252-273.

13. G.R. Liu, T.T. Nguyen, K.Y. Dai, K.Y. Lam, Theoretical aspects of the smoothed finite element method (SFEM), Int. J. Numer. Methods Eng. 71 (2007) 902-930.

14. N. Vu-Bac, H. Nguyen-Xuan, L. Chen, S. Bordas, P. Kerfriden, R.N. Simpson, G.R. Liu, T. Rabczuk, A node-based smoothed extended finite element method (NS-XFEM) for fracture analysis, Comput. Model. Eng. Sci 73(4) (2011) $331-355$.

15. L. Chen, T. Rabczuk, S.P.A. Bordas, G.R. Liu, K.Y. Zeng, P. Kerfriden, Extended finite element method with edge-based strain smoothing (ESm-XFEM) for linear elastic crack growth, Comput. Methods Appl. Mech. Eng. 209-212 (2012) $250-265$.

16. N. Vu-Bac, H. Nugyen-Xuan, L. Chen, C.K. Lee, G. Zi, X. Zhuang, G.R. Liu, T. Rabczuk, A phantom-node method with edge-based strain smoothing for linear elastic fracture mechanics, J. Appl. Math. http://dx.doi.org/10.1155/2013/978026. 
17. G.R. Liu, A generalized gradient smoothing technique and the smoothed bilinear form for Galerkin formulation of a wide class of computational methods, Int. J. Comput. Methods 5 (2) (2008) 199-236.

18. J.S. Chen, C.T. Wu, S. Yoon, Y. You, A stabilized conforming nodal integration for Galerkin mesh-free methods, Int. J. Numer. Methods Eng. 50 (2001) 435-466.

19. J.W. Yoo, B. Moran, J.S. Chen, Stabilized conforming nodal integration in the natural-element method, Int. J. Numer. Methods Eng. 60 (2004) 861-890.

20. T. Nguyen-Thoi, H.C. Vu-Do, T. Rabczuk, H. Nguyen-Xuan, A node-based smoothed finite element method (NS-FEM) for upper bound solution to visco-elastoplastic analyses of solids using triangular and tetrahedral meshes, Comput. Methods Appl. Mech. Eng. 199 (2010) 3005-3027.

21. T. Nugyen-Thoi, G.R. Liu, H. Nguyen-Xuan, C. Nguyen-Tran, Adaptive analysis using the node-based smoothed finite element method (NS-FEM), Int. J. Numer. Methods Biomed. Eng. 27 (2011) 198-218.

22. Q. Tang, G.Y. Zhang, G.R. Liu, Z.H. Zhong, Z.C. He, A three-dimensional adaptive analysis using the meshfree node-based smoothed point interpolation method (NS-PIM), Eng. Anal. Bound. Elements 35 (2011) 1123-1135.

23. S.C. Wu, G.R. Liu, H.O. Zhang, X. Xu, Z.R. Li, A node-based smoothed point interpolation method (NS-PIM) for three-dimensional heat transfer problems, Int. J. Therm. Sci. 48 (2009) 1367-1376.

24. G.R. Liu, L. Chen, T. Nguyen-Thoi, K.Y. Zeng, G.Y. Zhang, A novel singular node-based smoothed finite element method (NS-FEM) for upper bound 
solutions of fracture problems, Int. J. Numer. Methods Eng. 83 (2010) 1466-1497.

25. Z.Q. Zhang, G.R. Liu, Temporal stabilization of the node-based smoothed finite element method and solution bound of linear elastostatics and vibration problems, Comput. Mech. 46 (2010) 229-246.

26. S. Beissel, T. Belytschko, Nodal integration of the element-free Galerkin method, Comput. Methods Appl. Mech. Eng. 139 (1996) 49-74.

27. G.R. Liu, T. Nguyen-Thoi, K.Y. Lam, A novel alpha finite element method $(\alpha \mathrm{FEM})$ for exact solution to mechanics problems using triangular and tetrahedral elements, Comput. Methods Appl. Mech. Eng. 197 (2008) 3883-3897.

28. H. Nguyen-Xuan, T. Rabczuk, N. Nguyen-Thanh, T. Nguyen-Thoi, S. Bordas, A node-based smoothed finite element method with stabilized discrete shear gap technique for analysis of Reissner-Mindlin plates, Comput. Mech. 46 (2010) $679-701$

29. M.A. Puso, J.S. Chen, E. Zywicz, W. Elmer, Meshfree and finite element nodal integration methods, Int. J. Numer. Methods Eng. 74 (2008) 416-446.

30. T. Rabczuk, T. Belytschko, S.P. Xiao, Stable particle methods based on Lagrangian kernels, Comput. Methods Appl. Mech. Eng. 193 (2004) 1035-1063.

31. H. Feng, X.Y. Cui, G.Y. Li, A stable nodal integration method with strain gradient for static and dynamic analysis of solid mechanics, Eng. Anal. Bound. Elements 62 (2016) 78-92.

32. G. Wang, X.Y. Cui, H. Feng, G.Y. Li, A stable node-based smoothed finite 
element method for acoustic problems, Comput. Methods Appl. Mech. Eng. 297 (2015) 348-370.

33. N.Z. Lima, R. C. Mesquita, Face-based gradient smoothing point interpolation method applied to 3-D electromagnetics, IEEE Trans. Magn. 50 (2) (2014) $537-540$.

34. D. Soares, Time-domain electromagnetic wave propagation analysis by edge-based smoothed point interpolation methods, J. Comput. Phys. 234 (2013) $472-486$

35. F.G. Guimarães, R.R. Saldanha, R.C. Mesquita, D.A. Lowther, J.A. Ramírez, A meshless method for electromagnetic field computation based on the multiquadric technique, IEEE Trans. Magn. 43 (4) (2007) 1281-1284.

36. S.L. Ho, S. Yang, J.M. Machado, H.C. Wong, Application of a meshless method in electromagnetics, IEEE Trans. Magn. 37 (5) (2001) 3198-3202.

37. C. Hérault, Y. Maréchal, Boundary and interface conditions in meshless methods, IEEE Trans. Magn. 35 (3) (1999) 1450-1453.

38. Y. Maréchal, Some meshless methods for electromagnetic field computations, IEEE Trans. Magn. 34 (5) (1998) 3351-3354.

39. F.Z. Louaï, N. Naït-Saïd, S. Drid, Implementation of an efficient element-free Galerkin method for electromagnetic computation, Eng. Anal. Bound. Elements 31 (2007) 191-199.

40. X. Liu, B.-Z. Wang, S. Lai, Element-free Galerkin method in electromagnetic scattering field computation, J. Electromagn. Waves Appl. 21 (14) (2007) 
1915-1923.

41. L. Xuan, Z. Zeng, B. Shanker, L. Udpa, Element-free Galerkin method for static and quasi-static electromagnetic field computation, IEEE Trans. Magn. 40 (1) (2004) 12-20.

42. K. Fujiwara, T. Nakata, Results for benchmark Problem 7 (Asymmetrical conductor with a hole) COMPEL-Int. J. Comput. Math. Electr. Electron. Eng. 9 (3) (1990) 137-154.

43. H. Tsuboi. M. Tanaka, K. Ikeda, K, Nishimura, Computation results of the TEAM workshop Problem 7 by finite element methods using tetrahedral and hexahedral elements, J. Mater. Process. Technol. 108 (2001) 237-240.

44. T. Nakata, N. Takahashi, K. Fujiwara, Y. Shiraki, Comparison of different finite elements for 3-D eddy current analysis, IEEE Trans. Magn. 26 (2) (1990) 434-437.

45. C. Anitescu, Y. Jia, Y.J. Zhang, T. Rabczuk, An isogeometric collocation method using superconvergent points, Comput. Methods Appl. Mech. Eng. 284 (2015) 1073-1097. 